\title{
Influencia de la responsabilidad social empresarial en la imagen corporativa de los clientes de La Posada del Mirador, en Barranco ${ }^{1}$
}

\section{Influence of corporate social responsibility on the corporate image of customers of La Posada del Mirador in Barranco}

\author{
Frida Luperdi Cárdenas* \\ Escuela Profesional de Ciencias de la Comunicación, \\ Universidad de San Martín de Porres, Perú
}

\section{Resumen}

La responsabilidad social empresarial (RSE) en la actualidad genera innovación, transparencia, eficiencia, desarrollo confianza, reputación, y ello sirve para que la empresa tenga una mayor competitividad y así tenga una diferenciación con las demás empresas, esto genera valor a la organización.

La presente investigación tiene el objetivo principal de identificar cómo influye la RSE en la imagen corporativa del servicio de expendio de comidas La Posada del Mirador, del distrito de Barranco, año 2017, en sus principales públicos externos.

Se concluye que la RSE influye significativamente en la imagen corporativa del servicio de expendio de comidas La Posada del Mirador.

Palabras clave: responsabilidad social, imagen corporativa, identidad, reputación corporativa, cultura organizacional, cultura de confianza.

Este es un artículo Open Access bajo la licencia Creative 


\begin{abstract}
Corporate social responsibility currently generates innovation, transparency, efficiency, confidence and reputation, which help companies to become more competitive and thus differentiate themselves from other companies, and create value for the organization.
\end{abstract}

This research mainly aims to identify how corporate social responsibility influenced corporate image in food service - La Posada del Mirador - and its main external audiences in the district of Barranco in the year 2017.

Consequently, it is confirmed that social responsibility significantly influences corporate image in food service - La Posada del Mirador.

Keywords: social responsibility, corporate image, identity, corporate reputation, organizational culture, culture of trust.

\title{
Introducción
}

En el presente estudio referido a la responsabilidad social empresarial (RSE) nos hemos planteado los objetivos de identificar la influencia de la responsabilidad política en la identidad cultural; determinar cómo influye la responsabilidad económica en la identidad visual; y establecer la influencia de la responsabilidad ética en la reputación corporativa.

Consideramos que esta investigación cuantitativa es importante, en tanto, nos permite evidenciar la relevancia de la responsabilidad social en toda organización. Los resultados pretenden contribuir en el ámbito teórico práctico, considerando las principales teorías de las Escuelas Latinoamericana, Europea y Norteamericana y el aporte de grandes autores sobre la influencia de la responsabilidad social empresarial en la imagen corporativa dentro de una organización, sea esta pública o privada. 


\section{Responsabilidad social empresarial (RSE)}

La RSE como función de las relaciones públicas, en la actualidad, toma un alto nivel de importancia, es por eso, que en los últimos años ha sido materia de estudio de diferentes académicos, ya que surge como una preocupación del empresario por su entorno permitiendo al profesional de las relaciones públicas conseguir una mejor imagen para la organización con sus públicos. Exhorta a las organizaciones a ser capaces de asumir su compromiso con la sociedad y el país, debido a que toda organización está conformada por hombres para hombres.

La construcción de una reputación, empieza desde el primer contacto, a través de una imagen transparente, donde se da los primeros pasos para lograr una identidad corporativa. Sin la debida atención y capacitación continua a los stakeholders, no se llega a formar un conjunto de valores que establezcan una percepción positiva a un mediano y largo plazo (Illescas, 1995).

Es por lo tanto, importante tener una práctica de la responsabilidad social empresarial dentro de las organizaciones, es la base para la buena relación entre la institución pública o privada y el nivel de aceptación que puede tener entre sus públicos (Grunig \& Hunt, 2000).

Toda organización tiene una responsabilidad social como generadoras de riqueza con la sociedad, es decir, toda organización debe ser responsable en el desarrollo del país donde se encuentre operando, en la creación de empleo y su contribución tributaria para poder generar propuestas de políticas públicas que contribuyan con el crecimiento de la sociedad (Ferrari \& França, 2012).

No existe una responsabilidad social, sin primero pasar por el cumplimiento integral de las normas del país de origen de la empresa, siendo el punto de partida para apoyar al crecimiento del mismo; sin embargo, el fin de un acto socialmente responsable no son acciones realizadas para una inversión social (Norero, 2000).

Para realizar acciones socialmente responsables, se debe permitir el cumplimiento de un plan estratégico basado en la misión y visión con la que surgió la empresa (Ferrari \& França, 2012). 
Las tácticas de responsabilidad social son desarrolladas basadas en la filosofía empresarial, por ello son voluntarias, mostrando no solo la ética sino el compromiso social con todos los públicos, pero tiene que partir de la alta gerencia para su consolidación en la organización.

La RSE, es entonces, aquella decisión que toma la organización para establecer una relación en búsqueda del bienestar social y natural. Esta relación que crea y mantiene es dirigida a todos los públicos de interés, llamados también stakeholders, la cual debe velar por el equilibrio entre los intereses de la organización, de los públicos y la preservación del medioambiente.

La RSE es una tendencia dentro del mundo globalizado, la cual exige a las organizaciones una relación responsable con sus públicos de interés, sean estos públicos internos o externos. Es por ello que una de las acciones iniciales es la creación de una marca de fácil reconocimiento para sostener una imagen corporativa sólida. La RSE rara vez se ve reflejada en la cultura y estructura de la organización, y generalmente se la conceptualiza casi como la imagen, marca y reputación. Estos tres conceptos son muy importantes, pero según mi lectura de investigación, se alinean cuando el programa de RSE es central para la organización y sus esfuerzos lo hacen con las metas de la institución.

Para Carroll (1979), la responsabilidad social debe ser articulada e interrelacionada en:

1. Una definición básica de responsabilidad social (es decir, inuestra responsabilidad va más allá de las preocupaciones económicas y legales?) 2. Una enumeración de las cuestiones para las que existe una responsabilidad social (es decir, ¿cuáles son las áreas sociales -ambientales, seguridad del producto, discriminación, etc.- en las que se tiene una responsabilidad?). 3. Una especificación de la filosofía de respuesta (es decir, ireaccionan a los problemas o son proactivos?). Cada una de estas necesidades necesita de elaboración. Por ello, para que una definición de responsabilidad social aborde plenamente toda la gama de obligaciones que el negocio tiene para la sociedad, debe incluir las categorías económicas, legales, éticas y discrecionales del desempeño empresarial. (p. 499) 
Es por ello que tomaremos en cuenta el concepto de responsabilidad social de Carroll (1979), en La Posada del Mirador, analizando si la responsabilidad social del servicio de expendio de comidas va más allá de las preocupaciones económicas, legales y éticas. Así como también si cuenta con áreas sociales y ambientales, y por último, si son proactivos en su gestión.

La responsabilidad social actual se entiende como el diagnostico de desarrollo de diferentes niveles, donde se aplica dicha responsabilidad en las organizaciones, empresas privadas e individuos o familiares en relación con sus respectivos stakeholders; no son ordenes expeditas de cumplimiento, sino un compromiso interno desde la alta dirección, hasta las diversas ramas del organigrama institucional, que se vuelve en acciones con significado lógico y de perspectivas positivas (Caravedo, 2002).

\section{Responsabilidad politica}

Para definir el concepto de responsabilidad política, es necesario entender el concepto mismo de política. Se puede decir que es el arte de reflexionar también sobre la conveniencia de las acciones o decisiones que afectan la vida en común.

La política está basada en el concepto mismo de la empresa, ya que son las estrategias planteadas para seguir los procedimientos reglamentados y así llegar a los objetivos planteados a corto, mediano y largo plazo.

Si bien la RSE cumple en contribuir en los relacionamientos de la empresa con sus públicos, es necesario poder abordar la política de la organización.

Esta responsabilidad social basada en la política social, está orientada hacia el establecimiento definido de la misión de la empresa, entendiendo una construcción de acciones significativas dentro de la organización como equipo.

De esta forma, encontramos que una responsabilidad política va de la mano con los valores institucionales que se manejan dentro de la empresa, los principales inconvenientes surgen, cuando se deja de cumplir las leyes y normas nacionales por beneficios económicos (responsabilidad fiscal) 
dejando de lado el primer escalón de toda empresa socialmente responsable. Rompiendo con la transparencia, perdiendo la credibilidad y confianza de los stakeholders.

\section{Responsabilidad económica}

El ámbito económico de una empresa es sin duda una de los apartados más importantes, ya que es el eje por el cual una empresa busca crecer dentro del mercado. La rentabilidad de la misma se produce gracias a una serie de políticas económicas.

El pilar de la responsabilidad económica es conseguir el crecimiento de los ingresos, para poder distribuirlo y se pueda invertir de forma favorable para la empresa (Almagro, Garmedia, \& De la Torre, 2010).

Sin embargo, también existe una economía social. Almagro et al. (2010) afirman que «la economía social es pionera en la aplicación del principio de responsabilidad social toda vez que dicho principio forma parte principal de los objetivos estratégicos de las entidades que integran este sector institucional del sistema económico» (p. 134).

Si los ingresos de la empresa son invertidos, en función de sus objetivos dentro del plan estratégico para cumplir con metas definidas en un cierto tiempo, se puede decir que es socialmente responsable.

\section{Responsabilidad ética}

Para concebir la responsabilidad ética, primero se debe conceptualizar la ética como parte del desarrollo del hombre. Leuridan (2016), sostiene que la ética de la vida «es una filosofía que reflexiona sobre el arte de vivir» (p. 130).

La ética «se aplica a la conducta presuntamente libre y responsable de una persona, cuando una conducta es juzgada por los demás como adecuada a un ser humano, como digna de alabanza, merecedora de imitación y como deseable» (Cobo, 2001, p. 25). 
Se demuestra entonces que la ética es parte del compromiso interno de la persona que se debe explorar y construir para generar la tendencia de ser responsables con el ambiente, la sociedad y la economía no solo emocional, sino también lógica, como se dice un arte de saber cómo desarrollarse en la vida misma.

Carroll (1979) nos dice que:

Aunque las dos primeras categorías (responsabilidades económicas y legales) incorporan normas éticas, existen comportamientos y actividades adicionales que no necesariamente se codifican en leyes, pero que, sin embargo, se esperan de las empresas. En los últimos años, sin embargo, las responsabilidades éticas han sido claramente subrayadas, aunque el debate continúa sobre lo que es y no es ético. Basta con decir que la sociedad tiene expectativas de negocio más allá de los requisitos legales. (p. 500)

Carroll (1979) es muy puntual respecto a la ética, menciona que debe estar presente en todos los niveles de la organización y es una de las características integrales de una organización que es socialmente responsable, a pesar que no existe un concepto aceptado internacionalmente, es tener disciplina en realizar cualquier práctica que al cumplir objetivos claros cumple con una perspectiva de valores sociales.

La inclinación entre el bien y el mal, que está expuesta diariamente conlleva a la saturación excesiva de la información, por lo cual se debe ser selectivo entre las herramientas y objetivos que incluyan la razón y la libertad del hombre (Moscol, 1990).

Por ello, la ética de las relaciones públicas no solo se basa en la buena voluntad o intención pura, sino en la consistencia del cumplimiento de las políticas y valores del deber, y sus consecuencias con los derechos de los stakeholder para proteger la dignidad humana de los mismos.

En el ámbito de restaurantes de expendio de comida, el concepto de la RSE es visto de manera ordenada o metodológica, normalmente se tienen procedimientos de actuación ética social que se emplean de forma práctica, 
pero que no lleva ningún perfil de acción para aprovechar esa responsabilidad en favor de los mismos restaurantes impactando efectivamente al medio que rodea a la empresa. En el Perú, se observa a la RSE como un concepto y práctica nueva en búsqueda de la rentabilidad económica sin establecer un impacto negativo al entorno, cuidando el mantenimiento y respeto hacia los recursos naturales y medios sociales, donde tanto empresarios como los clientes del servicio de expendio puedan ofrecer y utilizar de manera responsable y sostenible.

\section{Imagen corporativa}

En los últimos años, la imagen es uno de los aspectos a considerar y proteger en las empresas, y se ha vuelto parte importante de las organizaciones.

Uno de los principales valores diferenciadores frente a otras organizaciones es la imagen corporativa y esta variable es el resultado de la proyección de la cultura organizacional.

Por este motivo, la imagen es una construcción en la identificación de una empresa. Es decir, del desarrollo constante de una cultura organizacional, donde cada colaborador interiorice que cada acción que desarrolla afecta a la empresa de forma directa e indirecta. Al reconocer el papel que se desarrolla, por ende, se concibe que el público externo perciba ese comportamiento (Pérez \& Solórzano, 1999).

Cada objetivo que se imponga la organización debe estar internalizado en el público interno, ya que con esto se definen los posibles alcances de éxitos de los mismos, porque la eficiencia y eficacia que se lleva acabo se ve reflejada diariamente (Capriotti, 1992).

La imagen corporativa es considerada el resultado claro de la interacción de todas las experiencias, creencias, conocimientos, emociones e impresiones que la gente tiene respecto a una empresa; es la idea de que no solo se trata de una acción en particular, sino de la respuesta a las acciones integrales, que se plasman en un plan estratégico para llegar a generar empatía y de esta forma generar vínculos con el público externo (Scheinsohn, 2009). 
Pintado y Sánchez (2013) señalan que:

El estudio de la imagen es un análisis complejo ya que trata de medir y analizar, no solo aspectos o características tangibles sino, atributos intangibles y, además, se hablan de atributos múltiples que son los que conforman la representación mental de la empresa o marca. Los dos grandes tipos de técnicas que se utilizan para estudiar la imagen corporativa son las técnicas cualitativas y cuantitativas. (p. 81)

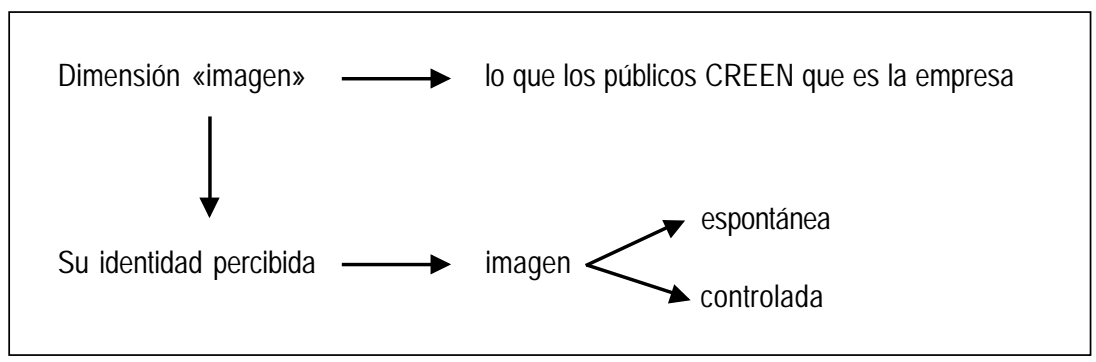

Figura 1. Creación de valor de la imagen corporativa. Adaptado de «Imagen corporativa: Influencia en la gestión empresarial» por Pintado y Sánchez, 2013.

En la Figura 1 se observa como esta dimensión de imagen debe sentirse de forma espontánea, pero debe hacerse seguimiento ya que representa la identidad que percibe el público externo.

Se vislumbra, que es un componente invaluable dentro de la organización, de la misma forma se puede medir, por lo tanto, se puede recoger resultados del cumplimiento de objetivos y aceptación social de la empresa en un mediano y largo plazo para modificar o seguir con el plan estratégico.

Al hablar de organización se abordan conceptos vinculantes o dependientes los unos de los otros como: imagen corporativa, identidad cultural, identidad visual y reputación corporativa, teniendo la primera relación con el grado de reconocimiento e importancia en las empresas. Este reconocimiento es ocasionado por su participación directa en el logro de los objetivos de la organización, pública o privada. 
Es por eso que la imagen corporativa de una empresa debe trabajar con un plan que identifique a la sociedad como un público significativo, evidenciando que la acción de la RSE beneficia no solo a la sociedad sino también a la organización, ya que su comportamiento comprometido con la sociedad proyecta indiscutiblemente una imagen positiva en sus públicos.

\section{Identidad cultural}

La identidad cultural no solo se estudia en un contexto social, sino también de la comunicación empresarial, ya que marca las pautas de equilibrio entre lo que es aceptado y discutible, además de comportamientos establecidos por paradigmas sociales que influyen en el proceso de imagen y reputación de una organización.

Así mismo, se entiende que la cultura involucra todo un conjunto de concepciones sociales que se aplican en la sociedad, en la cual la empresa se quiere posicionar; por ello, se debe realizar un estudio sobre qué valores y costumbres se pueden aplicar para ser aceptados dentro de la misma.

La identidad cultural es la expresión de la cultura organizacional, y es observable en la respuesta del público al momento de adquirir un producto o servicio de una organización, estas acciones son una aplicación constante de valores sociales que se desarrollan en la misma organización y es perceptible en el público externo (Ramos, 2008).

Actualmente, se lleva un control al momento de contratar un nuevo colaborador. Se busca personas que tengas valores afines a la empresa $u$ organización, para que se pueda desempeñar con más eficacia al momento de lograr los objetivos solicitados, perteneciendo al área de atención al cliente principalmente (Villafañe, 2000).

\section{Cultura de la confianza}

La confianza es una característica dentro de la empresa que se desarrolla de forma diaria dentro de las actividades de cualquier organización. A través del tiempo, esto genera el valor de confiabilidad que toda organización quiere tener como marca. 
Tener confianza significa que en cualquier crisis se debe actuar de forma transparente, teniendo un primer respaldo de los stakeholder de una organización. Solórzano y Pérez (1999) añaden: «Confianza, relacional mutua, apoyada por información con credibilidad que da acceso al conocimiento y legitimada por un nivel moral de comportamiento» (p. 20).

Desempeñar el uso adecuado de la confiabilidad es realizar un trabajo eficiente dentro de la organización, ya que se cumple el desarrollo óptimo de lo que se ha solicitado con las herramientas brindadas. Permitiendo que se vea reflejado en la calidad del producto o servicio ofrecido.

\section{Cultura organizacional}

Se refiere a los mensajes que utilizan todos los colaboradores de la organización en diversos niveles, entre ellos. Permitiendo así una contribución activa de todas las personas que pertenecen a una organización, abriendo una comunicación abierta flexible y multidireccional, involucrando de esta manera una comunicación estratégica que puede ser diseñada para alcanzar los objetivos propuestos.

\section{Identidad visual}

Una de las principales fuentes de personalización que se observa en la empresa, es la identidad visual, ya que es la primera impresión que se lleva el público externo con la empresa.

Para trabajar la identidad visual, es importante definir cuáles son las características principales de nuestra organización, con el fin de plantear no solo los colores ideales sino las formas con las que se va a diseñar la forma física de la marca, además de cómo se debe aplicar la identidad en todas las plataformas visuales, permitiendo la distinción de la misma en el mercado.

La identidad visual, al ser parte imprescindible de la identificación de la organización, es importante que se reconozca como un agente externo y que se desarrolle dentro del campo asignado para la identificación, también del público interno, ya que es el símbolo de la organización (Villafañe, 2000). 
De esta forma, podemos fundamentar que todo comunica, de forma más precisa de manera visual, que conlleva una unidad si se aplica correctamente, planificando a través de un proceso de una expresión visual, permitiendo captar la identidad de la empresa.

Se puede observar en el logotipo de La posada del Mirador las características que el color marrón representa para su empresa y que cumple como forma de integrar el confort con el lado rústico.

\section{Reputación corporativa}

La creación de valor en la reputación corporativa es una construcción social, que se espera consolidar a través del tiempo, permitiendo una estabilidad en la legitimidad de sus acciones y objetivos.

Este compromiso asumido por la empresa en seguir ciertas conductas y valores, dentro de su desarrollo debe estar complementado con el mismo comportamiento de su público interno, que se manifiesta en todo el ciclo del servicio de la empresa.

Por este motivo, la reputación corporativa es el juicio de valor al que toda organización está expuesta, donde cada stakeholder se ve afectado y afecta de una u otra forma, pero constituye la relación formal donde se intensifica la percepción y compromisos entre ellos (Menguzzato, 2009).

Uno de los valores que toda organización desea conseguir es una buena reputación, por ello, se trabaja diferentes acciones sociales, con el fin de agradar al público externo. Pero, olvidan que la reputación surge a través de un proceso continuo de trato con todos los stakeholder, no solo con los clientes o a través de acciones benéficas.

La reputación se trabaja de forma continua por la aceptación de los stakeholder, y como señala Martín (2008, p. 31) «constituye el resultado de un proceso de legitimación en el que determinados agentes externos e internos evalúan múltiples aspectos de la empresa» para generar rentas a través de su conexión con la imagen corporativa, permitiendo una valoración de la empresa y un acercamiento directo a la misma. 
Se puede decir que la reputación es el afecto que tienen los diferentes stakeholders a través del cumplimiento de percepciones que tuvieron al iniciar y mantener el contacto con la organización, esta capacidad para brindar el valor adecuado a cada grupo de interés lleva a brindarles lo que necesita y requiere. Por este motivo, esta característica es observable en un mediano a largo plazo.

\section{Diseño metodológico}

\section{Metodología, participantes}

Se utilizó el diseño no experimental, puesto que se realiza sin manipular, se trata de prestar atención al problema y compararlo con la realidad para comprobarlo. Esta investigación es transversal porque el instrumento se aplicó en una sola ocasión. Se desarrolló el siguiente esquema:

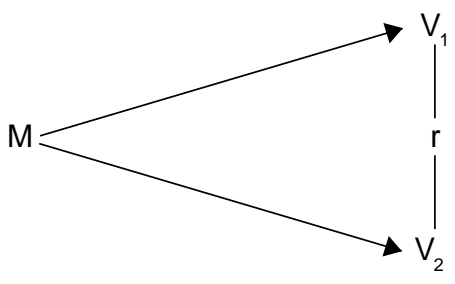

Donde:

$\mathbf{V}_{1}$ Responsabilidad social

$\mathrm{V}_{2}$ Imagen corporativa

r Correlación entre las variables de estudio

M Muestra

Aplicativa, ya que se tomaron conocimientos ya existentes como teorías, enfoques y principios en cada variable de estudio.

Explicativo, de carácter evaluativo, debido a que el trabajo se enfocó principalmente en la aplicación de responsabilidad social de la imagen corporativa-servicio de expendio de comidas de La Posada del Mirador en el distrito de Barranco, de la ciudad de Lima, a efectos de generar una evaluación concreta al apreciar cómo se regula la responsabilidad social en el rubro alimenticio, considerando que se trata de un área muy sensible. En este caso, la respuesta del consumidor es de carácter inmediato. 
La población la integraron personas adultas del distrito de Barranco. La muestra fue de 102 personas: 57 hombres y 45 mujeres, con edades de 25 a 55 años; aproximadamente $40 \%$ eran clientes asiduos al restaurante.

El análisis cuantitativo se realizó a partir de las encuestas aplicadas siendo procesadas mediante el ingreso de los datos en plantillas elaboradas en MS Excel para emitir reportes gráficos de los resultados.

\section{Instrumento}

Consistió en un cuestionario, cuya confiabilidad fue verificada con el valor del coeficiente correlacional de Pearson a través del programa estadístico SPSS-24.

\section{Resultados}

Se determina que la responsabilidad social empresarial influye significativamente en la imagen corporativa del público externo en el expendio de comida La Posada del Mirador del distrito de Barranco, año 2017. Asimismo, la responsabilidad política influye en la imagen cultural; la responsabilidad económica influye en la identidad visual; y la responsabilidad ética influye en la reputación corporativa.

Tenemos que el valor del coeficiente correlacional de Pearson es 0.343 mayor que 0.2 , por lo tanto, existe una correlación positiva de $34.3 \%$ entre la variable responsabilidad social e imagen corporativa. El valor del coeficiente correlacional de Pearson es 0.364 mayor que 0.2 , por lo tanto, existe una correlación positiva de $34.6 \%$ entre la variable responsabilidad política e identidad cultural. El valor del coeficiente correlacional de Pearson es 0.321 mayor que 0.2 , por lo tanto, existe una correlación positiva de $33.1 \%$ entre la variable responsabilidad económica e identidad visual. Por último, el valor del coeficiente correlacional de Pearson es 0.364 mayor que 0.2 , por lo tanto, existe una correlación positiva de $36.40 \%$ entre la variable responsabilidad ética y reputación corporativa.

Los resultados obtenidos han sido agrupados y planteados como se detallan a continuación. 
Tabla 1

Instrumento de medición de la responsabilidad social empresarial y la imagen corporativa

\begin{tabular}{|c|c|c|c|c|}
\hline Indicadores & Preguntas & Indeciso & $\begin{array}{l}\text { Parcialmente } \\
\text { de Acuerdo }\end{array}$ & $\begin{array}{l}\text { Totalmente de } \\
\text { Acuerdo }\end{array}$ \\
\hline \multirow{2}{*}{ Normas } & $\begin{array}{l}\text { 1. ¿Considera usted que La Posada del } \\
\text { Mirador cumple con las normas de } \\
\text { salubridad? }\end{array}$ & $8.8 \%$ & $78.4 \%$ & $12.7 \%$ \\
\hline & $\begin{array}{l}\text { 2. ¿Concuerda en afirmar que La Posada del } \\
\text { Mirador cumple con las normas de } \\
\text { seguridad? }\end{array}$ & $6.9 \%$ & $77.5 \%$ & $15.7 \%$ \\
\hline \multirow[t]{2}{*}{ Legitimidad } & $\begin{array}{l}\text { 3. ¿Considera usted que La Posada del } \\
\text { Mirador cuenta con licencia de } \\
\text { funcionamiento? }\end{array}$ & $7.8 \%$ & $65.7 \%$ & $26.5 \%$ \\
\hline & $\begin{array}{l}\text { 4. ¿Puede usted afirma que La Posada del } \\
\text { Mirador se encuentra legalmente registrada } \\
\text { en la Sunat? }\end{array}$ & $4.9 \%$ & $46.1 \%$ & $49.0 \%$ \\
\hline \multirow[t]{2}{*}{ Credibilidad } & $\begin{array}{l}\text { 5. ¿Concuerda en afirmar que La Posada del } \\
\text { Mirador cumple con entregar boletas de } \\
\text { ventas y/o factura al término del consumo } \\
\text { realizado? }\end{array}$ & $6.9 \%$ & $60.8 \%$ & $32.4 \%$ \\
\hline & $\begin{array}{l}\text { 6. ¿Considera usted que La Posada del } \\
\text { Mirador ofrece un servicio de calidad? }\end{array}$ & $8.8 \%$ & $56.9 \%$ & $34.3 \%$ \\
\hline \multirow{2}{*}{ Inversión } & $\begin{array}{l}\text { 7. ¿Concuerda en afirmar que La Posada del } \\
\text { Mirador ha invertido en el último año en la } \\
\text { remodelación de su infraestructura? }\end{array}$ & $17.6 \%$ & $49.0 \%$ & $33.3 \%$ \\
\hline & $\begin{array}{l}\text { 8. ¿Puede usted afirmar que La Posada del } \\
\text { Mirador cuenta con equipos y materiales } \\
\text { para una buena atención? }\end{array}$ & $33.3 \%$ & $38.2 \%$ & $28.4 \%$ \\
\hline \multirow[t]{2}{*}{ Comportamientc } & $\begin{array}{l}\text { 9. ¿Puede usted afirmar que La Posada del } \\
\text { Mirador tiene un comportamiento } \\
\text { responsable frente a sus clientes?, por } \\
\text { ejemplo, calidad de productos, atención al } \\
\text { cliente, etc. }\end{array}$ & $27.5 \%$ & $42.2 \%$ & $30.4 \%$ \\
\hline & $\begin{array}{l}\text { 10. ¿Concuerda en afirmar que La Posada del } \\
\text { Mirador cumple con un comportamiento } \\
\text { responsable frente a sus trabajadores?, por } \\
\text { ejemplo, capacitaciones, buena presencia, } \\
\text { etc. }\end{array}$ & $6.9 \%$ & $55.9 \%$ & $37.3 \%$ \\
\hline
\end{tabular}




\begin{tabular}{|c|c|c|c|c|}
\hline Indicadores & Preguntas & Indeciso & $\begin{array}{l}\text { Parcialmente } \\
\text { de acuerdo }\end{array}$ & $\begin{array}{l}\text { Totalmente de } \\
\text { acuerdo }\end{array}$ \\
\hline \multirow{3}{*}{ Compromiso } & $\begin{array}{l}\text { 11. ¿Considera usted que La Posada del } \\
\text { Mirador cumple con lo que ofrece? }\end{array}$ & $6.9 \%$ & $50.0 \%$ & $43.1 \%$ \\
\hline & $\begin{array}{l}\text { 12. ¿Puede usted afirmar que el servicio } \\
\text { ofrecido en La Posada del Mirador } \\
\text { cumplió con sus expectativas? }\end{array}$ & $11.8 \%$ & $46.1 \%$ & $42.2 \%$ \\
\hline & $\begin{array}{l}\text { 13. ¿Considera usted que La Posada del } \\
\text { Mirador atendió o satisfizo sus } \\
\text { necesidades como cliente? }\end{array}$ & $3.9 \%$ & $52.0 \%$ & $44.1 \%$ \\
\hline $\begin{array}{l}\text { Cultura de } \\
\text { confianza }\end{array}$ & $\begin{array}{l}\text { 14. ¿Puede usted afirmar que confía en la } \\
\text { atención de los trabajadores de La } \\
\text { Posada del Mirador? }\end{array}$ & $2.0 \%$ & $54.9 \%$ & $43.1 \%$ \\
\hline \multirow{2}{*}{$\begin{array}{l}\text { Cultura } \\
\text { organizacional }\end{array}$} & $\begin{array}{l}\text { 15. ¿Concuerda en afirmar usted que los } \\
\text { trabajadores de La Posada del Mirador } \\
\text { expresan gentileza en su atención? }\end{array}$ & $6.9 \%$ & $55.9 \%$ & $37.3 \%$ \\
\hline & $\begin{array}{l}\text { 16. ¿Considera usted que los trabajadores } \\
\text { de La Posada del Mirador expresan } \\
\text { dedicación y pasión en su atención? }\end{array}$ & $15.7 \%$ & $53.9 \%$ & $30.4 \%$ \\
\hline \multirow[t]{2}{*}{ Logotipo } & $\begin{array}{l}\text { 17. ¿Puede usted afirmar que el logotipo de } \\
\text { La Posada del Mirador representa a un } \\
\text { establecimiento rustico? }\end{array}$ & $9.8 \%$ & $56.9 \%$ & $33.3 \%$ \\
\hline & $\begin{array}{l}\text { 18. ¿Considera usted que los colores del } \\
\text { logotipo de La Posada del Mirador } \\
\text { reflejan o manifiestan la experiencia } \\
\text { brindada? }\end{array}$ & $4.9 \%$ & $54.9 \%$ & $40.2 \%$ \\
\hline \multirow{2}{*}{$\begin{array}{l}\text { Notoriedad de } \\
\text { la marca }\end{array}$} & $\begin{array}{l}\text { 19. ¿Cree usted que es correcto afirmar que } \\
\text { La Posada del Mirador es uno de los } 10 \\
\text { restaurantes más importantes del distrito } \\
\text { de Barranco? }\end{array}$ & $5.9 \%$ & $50.0 \%$ & $44.1 \%$ \\
\hline & $\begin{array}{l}\text { 20. ¿Considera usted que La Posada del } \\
\text { Mirador tiene una presencia activa en } \\
\text { redes sociales? }\end{array}$ & $2.0 \%$ & $45.1 \%$ & $52.9 \%$ \\
\hline
\end{tabular}

Nota: Elaboración propia. 


\section{Discusión}

En el caso del sector de la distribución alimentaria en España las empresas buscan ventajas competitivas, mediante la responsabilidad social como atributo diferencial en la imagen corporativa, puesto que el manejo de la economía es más dinámica y su filosofía empresarial se convierte en un elemento integrador para la empresa (Saiz, 2013).

Las entidades de crédito españolas son responsables socialmente, porque analizan en detalle la importancia que asignan a sus diferentes grupos de interés la integración tales como: accionistas, empleados, clientes, proveedores, la comunidad y la administración pública.

\section{Referencia}

Almagro, J. J., Garmedia, J. A., \& De la Torre, I. (2010). Responsabilidad social: una reflexión global sobre la RSE. Madrid: Prentice Hall - Financial Times.

Capriotti, P. (1992). La imagen de la empresa. Estrategia para una comunicación integrada. Barcelona: Consejo Superior de Relaciones Públicas de España.

Caravedo, B. (2002). Cambio de sentido: una perspectiva para el desarrollo sostenible. Lima: Centro de Investigación de la Universidad del Pacífico.

Carroll, A. B. (1979). Un modelo conceptual tridimensional de desempeño corporativo. Academia de gestión, 4(1), 499.

Cobo, J. M. (2001). Ética profesional en ciencias humanas y sociales. Madrid: Huerga y Fierro editores, S. L.

Ferrari, M. A., \& França, F. (2012). Relaciones Públicas para el éxito de las organizaciones. Lima: Universidad de San Martín de Porres.

Grunig, J., \& Hunt, T. (2000). Dirección de relaciones públicas. Barcelona: Ediciones Gestión 2000.

Illescas, W. (1995). Cómo planear las Relaciones Públicas. Buenos Aires: Macchi Grupo Editor S. A.

Leuridan, J. (2016). El sentido de las dimensiones éticas de la vida. Lima: Universidad de San Martín de Porres.

Martín, G. (2008). Reputación empresarial y ventaja competitiva. Madrid: ESIC Editorial.

Menguzzato, M. (2009). La dirección de empresas ante los retos del siglo XXI. Valencia: Universidad de Valencia.

Moscol, R. E. (1990). Sesión Relaciones Públicas. Lima: Universidad de Piura.

Norero, A. (2000). Responsabilidad social y relaciones públicas. Lima, Perú: Universidad de San Martín de Porres.

Pérez, R., \& Solórzano, E. (1999). Relaciones Públicas Superiores una nueva pedagogía. Lima: Universidad de San Martín de Porres.

Pintado, T., \& Sánchez, J. (2013). Imagen corporativa. Influencia en la gestión empresarial. Madrid: ESIC Editorial.

Ramos, F. (2008). El protocolo de empresa: Herramientas para crear valor. La Coruña: Netbiblo, S. L.

Saiz, E. (2013). La responsabilidad social como atributo diferencial en la imagen corporativa: el caso del sector de la distribución alimentaria en España (Tesis doctoral). Universitat Pompeu Fabram, Madrid, España. Recuperado de http://www.tdx.cat/bitstream/handle/10803/132693/tesl.pdf?sequence=1\&isAllowed=y 
Scheinsohn, D. (2009). Comunicación Estratégica. Management y fundamentos de la Imagen Corporativa. Buenos Aires: Ediciones Granica S. A.

Solórzano, E., \& Pérez, R. (1999). Relaciones públicas superiores: una nueva pedagogía. Lima: Universidad de San Martín de Porres.

Villafañe, J. (2000). Imagen Positiva, Gestión Estratégica de la Imagen de las empresas. Madrid: Ediciones Pirámide.

1 El presente artículo está basado en la investigación titulada "Influencia de la responsabilidad social empresarial en la imagen corporativa del servicio de expendio de comidas La Posada del Mirador, en sus principales públicos externos, distrito de Barranco, año 2017", sustentada en la Universidad de San Martín de Porres. 


\section{ANEXO}

\section{ENCUESTA}

El presente cuestionario tiene como objetivo identificar cómo influye la responsabilidad social empresarial en la imagen corporativa en el servicio de expendio de comidas La Posada del Mirador del distrito de Barranco, año 2017.

A continuación, se les presenta una serie de preguntas, de ellas seleccionen las respuestas que ustedes consideren correctas y las que se ajusten a la realidad.

Lea atentamente cada ítem y responda con sinceridad, recuerde que es una encuesta anónima.

\section{RESPONSABILIDAD SOCIAL EMPRESARIAL}

\section{Norma}

1. ¿Considera usted que La Posada del Mirador cumple con las normas de salubridad?

\begin{tabular}{|c|c|c|c|c|}
\hline $\begin{array}{c}\text { Completamente en } \\
\text { Desacuerdo }\end{array}$ & En Desacuerdo & Indeciso & $\begin{array}{c}\text { Parcialmente de } \\
\text { Acuerdo }\end{array}$ & $\begin{array}{c}\text { Totalmente de } \\
\text { Acuerdo }\end{array}$ \\
\hline & & & & \\
\hline
\end{tabular}

2. ¿Concuerda en afirmar que La Posada del Mirador cumple con las normas de seguridad?

\begin{tabular}{|c|c|c|c|c|}
\hline $\begin{array}{c}\text { Completamente en } \\
\text { Desacuerdo }\end{array}$ & En Desacuerdo & Indeciso & $\begin{array}{c}\text { Parcialmente de } \\
\text { Acuerdo }\end{array}$ & $\begin{array}{c}\text { Totalmente de } \\
\text { Acuerdo }\end{array}$ \\
\hline & & & & \\
\hline
\end{tabular}

\section{Legitimidad}

3. ¿Considera usted que La Posada del Mirador cuenta con licencia de funcionamiento?

\begin{tabular}{|c|c|c|c|c|}
\hline $\begin{array}{c}\text { Completamente en } \\
\text { Desacuerdo }\end{array}$ & En Desacuerdo & Indeciso & $\begin{array}{c}\text { Parcialmente de } \\
\text { Acuerdo }\end{array}$ & $\begin{array}{c}\text { Totalmente de } \\
\text { Acuerdo }\end{array}$ \\
\hline & & & & \\
\hline
\end{tabular}


4. ¿Puede usted afirma que La Posada del Mirador se encuentra legalmente registrada en la Sunat?

\begin{tabular}{|c|c|c|c|c|}
\hline $\begin{array}{c}\text { Completamente en } \\
\text { Desacuerdo }\end{array}$ & En Desacuerdo & Indeciso & $\begin{array}{c}\text { Parcialmente de } \\
\text { Acuerdo }\end{array}$ & $\begin{array}{c}\text { Totalmente de } \\
\text { Acuerdo }\end{array}$ \\
\hline & & & & \\
\hline
\end{tabular}

\section{Credibilidad}

5. ¿Concuerda en afirmar que La Posada del Mirador cumple con entrega boletas de ventas y/o factura al término del consumo realizado?

\begin{tabular}{|c|c|c|c|c|}
\hline $\begin{array}{c}\text { Completamente en } \\
\text { Desacuerdo }\end{array}$ & En Desacuerdo & Indeciso & $\begin{array}{c}\text { Parcialmente de } \\
\text { Acuerdo }\end{array}$ & $\begin{array}{c}\text { Totalmente de } \\
\text { Acuerdo }\end{array}$ \\
\hline & & & & \\
\hline
\end{tabular}

6. ¿Considera usted que La Posada del Mirador ofrece un servicio de calidad?

\begin{tabular}{|c|c|c|c|c|}
\hline $\begin{array}{c}\text { Completamente en } \\
\text { Desacuerdo }\end{array}$ & En Desacuerdo & Indeciso & $\begin{array}{c}\text { Parcialmente de } \\
\text { Acuerdo }\end{array}$ & $\begin{array}{c}\text { Totalmente de } \\
\text { Acuerdo }\end{array}$ \\
\hline & & & & \\
\hline
\end{tabular}

\section{Inversión}

7. ¿Concuerda en afirmar que La Posada del Mirador ha invertido en el último año en la remodelación de su infraestructura?

\begin{tabular}{|c|c|c|c|c|}
\hline $\begin{array}{c}\text { Completamente en } \\
\text { Desacuerdo }\end{array}$ & En Desacuerdo & Indeciso & $\begin{array}{c}\text { Parcialmente de } \\
\text { Acuerdo }\end{array}$ & $\begin{array}{c}\text { Totalmente de } \\
\text { Acuerdo }\end{array}$ \\
\hline & & & & \\
\hline
\end{tabular}

8. ¿Puede usted afirmar que La Posada del Mirador cuenta con equipos y materiales para una buena atención?

\begin{tabular}{|c|c|c|c|c|}
\hline $\begin{array}{c}\text { Completamente en } \\
\text { Desacuerdo }\end{array}$ & En Desacuerdo & Indeciso & $\begin{array}{c}\text { Parcialmente de } \\
\text { Acuerdo }\end{array}$ & $\begin{array}{c}\text { Totalmente de } \\
\text { Acuerdo }\end{array}$ \\
\hline & & & & \\
\hline
\end{tabular}




\section{Comportamiento}

9. ¿Puede usted afirmar que La Posada del Mirador tiene un comportamiento responsable frente a sus clientes?, por ejemplo, calidad de productos, atención al cliente, etc.

\begin{tabular}{|c|c|c|c|c|}
\hline $\begin{array}{c}\text { Completamente en } \\
\text { Desacuerdo }\end{array}$ & En Desacuerdo & Indeciso & $\begin{array}{c}\text { Parcialmente de } \\
\text { Acuerdo }\end{array}$ & $\begin{array}{c}\text { Totalmente de } \\
\text { Acuerdo }\end{array}$ \\
\hline & & & & \\
\hline
\end{tabular}

10. ¿Concuerda en afirmar que La Posada del Mirador cumple con un comportamiento responsable frente a sus trabajadores?, por ejemplo, capacitaciones, buena presencia, etc.

\begin{tabular}{|c|c|c|c|c|}
\hline $\begin{array}{c}\text { Completamente en } \\
\text { Desacuerdo }\end{array}$ & En Desacuerdo & Indeciso & $\begin{array}{c}\text { Parcialmente de } \\
\text { Acuerdo }\end{array}$ & $\begin{array}{c}\text { Totalmente de } \\
\text { Acuerdo }\end{array}$ \\
\hline & & & & \\
\hline
\end{tabular}

\section{Compromiso}

11. ¿Considera usted que La Posada del Mirador cumple con lo que ofrece?

\begin{tabular}{|c|c|c|c|c|}
\hline $\begin{array}{c}\text { Completamente en } \\
\text { Desacuerdo }\end{array}$ & En Desacuerdo & Indeciso & $\begin{array}{c}\text { Parcialmente de } \\
\text { Acuerdo }\end{array}$ & $\begin{array}{c}\text { Totalmente de } \\
\text { Acuerdo }\end{array}$ \\
\hline & & & & \\
\hline
\end{tabular}

12. ¿Puede usted afirmar que el servicio ofrecido en La Posada del Mirador cumplió con sus expectativas?

\begin{tabular}{|c|c|c|c|c|}
\hline $\begin{array}{c}\text { Completamente en } \\
\text { Desacuerdo }\end{array}$ & En Desacuerdo & Indeciso & $\begin{array}{c}\text { Parcialmente de } \\
\text { Acuerdo }\end{array}$ & $\begin{array}{c}\text { Totalmente de } \\
\text { Acuerdo }\end{array}$ \\
\hline & & & & \\
\hline
\end{tabular}

13. ¿Considera usted que La Posada del Mirador atendió o satisfizo sus necesidades como cliente?

\begin{tabular}{|c|c|c|c|c|}
\hline $\begin{array}{c}\text { Completamente en } \\
\text { Desacuerdo }\end{array}$ & En Desacuerdo & Indeciso & $\begin{array}{c}\text { Parcialmente de } \\
\text { Acuerdo }\end{array}$ & $\begin{array}{c}\text { Totalmente de } \\
\text { Acuerdo }\end{array}$ \\
\hline & & & & \\
\hline
\end{tabular}




\section{IMAGEN CORPORATIVA}

\section{Cultura de la confianza}

14. ¿Puede usted afirmar que confía en la atención de los trabajadores de La Posada del Mirador?

\begin{tabular}{|c|c|c|c|c|}
\hline $\begin{array}{c}\text { Completamente en } \\
\text { Desacuerdo }\end{array}$ & En Desacuerdo & Indeciso & $\begin{array}{c}\text { Parcialmente de } \\
\text { Acuerdo }\end{array}$ & $\begin{array}{c}\text { Totalmente de } \\
\text { Acuerdo }\end{array}$ \\
\hline & & & & \\
\hline
\end{tabular}

\section{Cultura organizacional}

15. ¿Concuerda en afirmar usted que los trabajadores de La Posada del Mirador expresan gentileza en su atención?

\begin{tabular}{|c|c|c|c|c|}
\hline $\begin{array}{c}\text { Completamente en } \\
\text { Desacuerdo }\end{array}$ & En Desacuerdo & Indeciso & $\begin{array}{c}\text { Parcialmente de } \\
\text { Acuerdo }\end{array}$ & $\begin{array}{c}\text { Totalmente de } \\
\text { Acuerdo }\end{array}$ \\
\hline & & & & \\
\hline
\end{tabular}

16. ¿Considera usted que los trabajadores de La Posada del Mirador expresan dedicación y pasión en su atención?

\begin{tabular}{|c|c|c|c|c|}
\hline $\begin{array}{c}\text { Completamente en } \\
\text { Desacuerdo }\end{array}$ & En Desacuerdo & Indeciso & $\begin{array}{c}\text { Parcialmente de } \\
\text { Acuerdo }\end{array}$ & $\begin{array}{c}\text { Totalmente de } \\
\text { Acuerdo }\end{array}$ \\
\hline & & & & \\
\hline
\end{tabular}

\section{Logotipo}

17. ¿Puede usted afirmar que el logotipo de La Posada del Mirador representa a un establecimiento rustico?

\begin{tabular}{|c|c|c|c|c|}
\hline $\begin{array}{c}\text { Completamente en } \\
\text { Desacuerdo }\end{array}$ & En Desacuerdo & Indeciso & $\begin{array}{c}\text { Parcialmente de } \\
\text { Acuerdo }\end{array}$ & $\begin{array}{c}\text { Totalmente de } \\
\text { Acuerdo }\end{array}$ \\
\hline & & & & \\
\hline
\end{tabular}

18. ¿Considera usted que los colores del logotipo de La Posada del Mirador reflejan o manifiestan la experiencia brindada?

\begin{tabular}{|c|c|c|c|c|}
\hline $\begin{array}{c}\text { Completamente en } \\
\text { Desacuerdo }\end{array}$ & En Desacuerdo & Indeciso & $\begin{array}{c}\text { Parcialmente de } \\
\text { Acuerdo }\end{array}$ & $\begin{array}{c}\text { Totalmente de } \\
\text { Acuerdo }\end{array}$ \\
\hline & & & & \\
\hline
\end{tabular}




\section{Notoriedad de la marca}

19. ¿Cree usted que es correcto afirmar que La Posada del Mirador es uno de los 10 restaurantes más importantes del distrito de Barranco?

\begin{tabular}{|c|c|c|c|c|}
\hline $\begin{array}{c}\text { Completamente en } \\
\text { Desacuerdo }\end{array}$ & En Desacuerdo & Indeciso & $\begin{array}{c}\text { Parcialmente de } \\
\text { Acuerdo }\end{array}$ & $\begin{array}{c}\text { Totalmente de } \\
\text { Acuerdo }\end{array}$ \\
\hline & & & & \\
\hline
\end{tabular}

20. ¿Considera usted que La Posada del Mirador tiene una presencia activa en redes sociales?

\begin{tabular}{|c|c|c|c|c|}
\hline $\begin{array}{c}\text { Completamente en } \\
\text { Desacuerdo }\end{array}$ & En Desacuerdo & Indeciso & $\begin{array}{c}\text { Parcialmente de } \\
\text { Acuerdo }\end{array}$ & $\begin{array}{c}\text { Totalmente de } \\
\text { Acuerdo }\end{array}$ \\
\hline & & & & \\
\hline
\end{tabular}

Gracias por su colaboración. 
